\title{
The use of extra-corporeal membrane oxygenation post-cardiotomy: the survival benefits
}

\author{
Tino Harahwa ${ }^{1}$, Cheryl Yan Ting Chor $^{1}$, and Amer Harky ${ }^{2}$ \\ ${ }^{1}$ St. George University London \\ ${ }^{2}$ Liverpool Heart and Chest Hospital NHS Foundation Trust
}

April 28, 2020

\begin{abstract}
Extra Corporeal Membrane Oxygenation (ECMO) is a supportive therapy used to provide cardiac support with or without respiratory support in the event of cardiopulmonary failure. The two main types of ECMO are Veno-arterial ECMO (VA-ECMO) and Veno-venous ECMO (VV-ECMO). The use of ECMO in cardiac surgery has been established in cases of post-cardiotomy cardiogenic shock which is refractory to conventional therapy with inotropes and intra-aortic balloon pulsation support. Survival for this, otherwise, fatal condition has been shown to be improving through the use of ECMO. However, the decision and timing to initiate ECMO therapy remains selective and is dependent on a range of factors such as patient factor, clinician's judgement, meaning there is no consistent and solid ground regarding the timing of ECMO initiation. This article will provide an extensive review of ECMO indications, contraindications, complications and outcomes to analyse the survival benefit of ECMO following cardiac surgery.
\end{abstract}

\section{Introduction}

Extra Corporeal Membrane Oxygenation (ECMO) is a form of mechanical circulatory support and extracorporeal life support system that provides cardiopulmonary support in patients with cardiac and/or pulmonary failure that is refractory to conventional medical management ${ }^{1,2}$. It is utilised to provide oxygenation, remove carbon dioxide and provide perfusion support via a circuit consisting of arterial and/or venous cannulae, connecting tubing, a blood pump and a gas exchange device. ECMO is broadly divided into two types: veno-arterial ECMO (VA-ECMO) and veno-venous-ECMO (VV-ECMO), which differ not only in their configurations and functions, but also their indications. VV-ECMO is indicated for pulmonary failure due to any cause or following lung transplantation due to primary graft failure whilst VA-ECMO is indicated for severe refractory cardiac failure due to any cause or for post-cardiotomy cardiogenic shock (PCCS) due to failure to wean from cardiopulmonary bypass $(\mathrm{CPB})$.

Institution of ECMO should only be on a temporary basis as a bridge to recovery, meaning until organ recovery occurs at which point ECMO can be removed ${ }^{1}$. ECMO may also be utilised as a bridge to destination therapy, meaning it may be used until implantation of a permanent ventricular assist device (VAD) or as a bridge to transplant therapy for use until organ transplantation is carried out. It is not useful in cases where pathology is not thought to be reversible and end organ functional recovery likely.

Whilst ECMO is a supportive therapy rather than a disease modifying treatment, it has been demonstrated to improve patient outcomes. The patient outcomes associated with the use of ECMO are dependent on the indication and the patient population it is utilised in with the survival to discharge rates for its use in acute respiratory failure being reported as ranging from $59 \%$ to $73 \%^{3}$. Survival to discharge for ECMO for cardiac support has been reported to be between $43 \%$ and $53 \%$. Survival to discharge for ECMO indicated for cardiopulmonary resuscitation has been reported as ranging from $29 \%$ to $42 \%$. In cardiac surgery patients 
specifically, various studies have demonstrated the survival benefit associated with institution of VA-ECMO in patients with PCCS which would otherwise be fatal ${ }^{4-13}$.

\section{Literature Search Method}

A comprehensive literature search is done through all the available electronic database to identity articles that discussed the use of extra-corporeal membrane oxygenation and mechanical circulatory support following cardiac surgery. The key words used were 'mechanical circulatory' 'extra-corporeal membrane oxygenation' 'ECMO 'Cardiac surgery' 'VA-ECMO OR VV-ECMO' 'Cardiogenic shock' 'Intra-aortic balloon pump' 'circulatory support' 'mechanical support'. Those key words were used in the form of combined search terms to maximize the search outputs. The inclusion criteria were articles that discussed the indications, outcomes and survival benefit from using mechanical circulatory support in the form of VV-ECMO or VA-ECMO, limits were placed on English language papers only. A summary table for each relevant section is provided, where applicable.

\section{Types of ECMO and Indication}

The two main subtypes of ECMO, VV-ECMO and VA-ECMO, differ in the configurations of their circuit which allows for them to carry out different functions and enables them to be utilised for respiratory support, cardiac support, or both ${ }^{1,2}$.

VV-ECMO involves venous cannulation alone- either single dual-lumen cannulation via the right jugular vein to enable removal of blood from the vena cava or right atrium by a pump device for oxygenation via an extracorporeal membrane oxygenator before it is returned to the right atrium, or double cannulation where blood is removed from the inferior vena cava (IVC) vein through cannulation of the right common femoral vein and infused into the right atrium via cannulation of the right internal jugular or vice versa though the former is more commonly used in modern practice (Figure 1) ${ }^{14}$. This configuration allows for gas exchange to occur but does not provide circulatory support, meaning VV-ECMO is only indicated for isolated respiratory failure and the patient must be haemodynamically stable. By contrast, VA-ECMO involves both arterial and venous cannulation, enabling removal of blood from the right atrium or IVC before it is returned to the arterial system via a peripheral cannula i.e. peripheral VA-ECMO (through the femoral, axillary or carotid artery) or a central cannula i.e. central VA-ECMO via the ascending aorta (Figure 2) ${ }^{15}$. The setup of the VA-ECMO circuit enables it to carry out gas exchange along with providing circulatory support thus it is indicated for isolated cardiac failure along with combined cardiopulmonary failure. The key differences between the two types of ECMO are summarised in Table 1.

Indications for ECMO are broadly divided according to cardiac support, respiratory support or a combination of both in the case of cardiopulmonary failure ${ }^{1}$ VA-EMCO is indicated for cardiac failure where there is a low cardiac output (defined as a cardiac index less than $2 \mathrm{~L} / \mathrm{min} / \mathrm{m}^{2}$ ) and hypotension (defined as a systolic blood pressure of less than $90 \mathrm{mmHg}$ ) refractory to adequate intravascular volume, high-dose inotropic support and IABP support ${ }^{16-18}$. Both VV- and VA-ECMO are indicated for acute respiratory failure as rescue therapy to provide gas exchange until the lungs recover or as a bridge to lung transplantation ${ }^{19-21}$. The main absolute contraindications to ECMO are pre-existing pathologies that make recovery unlikely such as disseminated malignancy and severe neurological injury, or unsuitability for destination therapy. Specific cardiac and respiratory indications, absolute and relative contraindications to for ECMO are summarised in Table 2.

\section{ECMO and coronary artery bypass grafting}

Post-cardiotomy cardiogenic shock (PCCS) has an incidence of 2-6\% in patients undergoing cardiac surgery and of these, an estimated $0.5-1 \%$ are refractory to maximal-dose inotropic support and IABP support ${ }^{4-6}$. Refractory PCCS rapidly leads to multi-organ failure and has an almost $100 \%$ mortality rate which makes it fatal without the use of MCS using devices such as VA-ECMO; thus, there is a clinical indication for its use in this cohort.

A range of retrospective studies have looked at outcomes including survival to weaning from ECMO and survival to hospital discharge for patients who have undergone cardiac surgery, but only a few of these have 
looked at outcomes in patients who underwent coronary artery bypass graft (CABG) surgery specifically ${ }^{6,9-11}$. The largest cohort of these studies analysed outcomes and survival in 517 patients treated with ECMO for PCCS, of which $37 \%$ had undergone isolated CABG and $16.8 \%$ CABG in combination with valve surgery ${ }^{6}$. The study results showed that undergoing isolated CABG was associated with better survival, with an inhospital mortality rate of $44 \%$ which was better when compared to that of other cardiac procedures $(95 \%$ Confidence Interval (CI) $0.29-0.68 ; P<0.001$ ). A similar association was shown in another study looking at 31 patients ( $28 \%$ of total cohort) who were treated with ECMO following isolated CABG which demonstrated that undergoing isolated CABG was associated with improved in-hospital mortality on EMCO when compared to other cardiac procedures $(41.3 \% \text { vs } 18.8 \%)^{9}$.

By contrast, a 2010 retrospective study looking at 233 patients who had undergone cardiac surgery and required ECMO post-operatively over an 11-year period, reported an increased hospital mortality which was associated with having undergone isolated CABG $(P=0.0015)$, among other factors ${ }^{10}$. This was also demonstrated in another retrospective study involving analysis of outcomes for 101 patients who received central VA-ECMO at a single centre over an 8-year period which reported the survival rate for isolated CABG as $17.2 \%$, a figure lower than the overall survival rate of $27.7 \%$ at 1-year follow-up and is lower than survival rates reported in similar studies ${ }^{11}$. Additionally, patients requiring VA-ECMO following isolated CABG had a significantly increased mortality risk (Odds Ratio (OR) 3.23; 95\% CI 1.1-9.4; $\mathrm{P}=0.021$ ) compared to patients who did not undergo coronary surgery. Outcomes in ECMO following CABG from the included studies are summarised in Table 3 .

Whilst there is no consensus on outcomes for patients requiring ECMO following isolated CABG, the aforementioned studies all demonstrated that in patients who had undergone CABG in combination with another cardiac procedure, namely valve surgery, was associated with a decreased survival rate with some studies quoting survival rates as low as $12.5 \%^{6,9-11}$.

Given that mortality rates for refractory PCCS without ECMO are almost always 100\% this demonstrated the survival benefit of ECMO in post-CABG patients even though reported survival rates are variable. None of the studies included looked at the effect of differing ECMO initiation times on mortality and morbidity rates making it difficult to ascertain the best timing for starting ECMO for patients undergoing CABG surgery. In the studies which reported survival rates greater 40\%, ECMO was instituted either intra-operatively during the primary cardiac procedure or within 24 hours following surgery ${ }^{6,9}$. Whilst in studies that reported lower survival rates, there was no standardisation for when ECMO was instituted with ECMO being used as late as after the sixth post-operative day or data for when ECMO was instituted was missing completely. This would seem to suggest that ECMO should be given within the first 24 hours to maximise the survival benefit, though more research analysing the effect of when to start ECMO is required going forward.

\section{ECMO and Valve surgeries}

Outcomes for patients requiring EMCO for PCCS refractory to maximal-inotropic support and IABP support in patients who have undergone valve surgery have been analysed across few studies ${ }^{6,22,23}$. Rastan et al ${ }^{6}$ carried out a study with a cohort of 517 patients who required ECMO for PCCS, of which $14.3 \%$ had undergone isolated valve surgery whilst $16.8 \%$ had undergone valve surgery in combination with CABG. This study found that post-operative ECMO requirement following mitral valve surgery was associated with a reduced survival rate when compared to other cardiac procedures (OR 2.08; 95\% CI 1.20-3.63;P=0.010). Similarly, Onorati et al ${ }^{22}$ found that in patients undergoing aortic valve replacement for aortic stenosis, post-operative ECMO was a predictor for early death as it was associated with a greater 30-day mortality rate (OR 9.8; 95\% CI 1.1-63.4; $P=0.029$ ) when compared to that of other cardiac procedures. However, this study has not yet reached its 5 -year endpoint, meaning the reported findings are only preliminary.

More recently, the institution of ECMO with a TandemHeart (TH) percutaneous Left VAD i.e. a THECMO, was found to provide stabilisation of patient haemodynamics and hypoxaemia in two patients with acute severe mitral regurgitation (MR) associated with cardiogenic failure and hypoxaemia ${ }^{23}$. Acute severe MR associated with cardiogenic shock and hypoxaemia can only be definitively managed with mitral valve 
surgery, but usually this surgery is associated with high peri-operative mortality rates of $25-46 \%$, a poor outcome due to patients being haemodynamically unstable and having refractory hypoxaemia. In this case series, the institution of TH-ECMO resulted in prompt stabilisation of the patients' haemodynamics and hypoxaemia which in turn allowed them to have early surgical intervention to manage their acute severe MR. This case series shows the potential survival benefit of ECMO for acute severe MR associated with cardiogenic shock and hypoxaemia. However, it is important to note that the study only reported on two cases making the sample size far too small for results to be statistically significant and the patients would need to be followed up for a lengthier period of time to ascertain the longer-term outcomes associated with ECMO use. Outcomes in patients who had ECMO having undergone valve surgery are summarised in Table 4.

Whilst post-operative requirement following valve surgery may be associated with worse prognosis when compared to people who did not require ECMO post-valve surgery, it again does improve survival for refractory PCCS when compared to without the use of MCS. It is unclear when the best time to institute ECMO in patients undergoing valve-surgery would be but based on the studies analysed, ECMO institution was associated with increased mortality when inserted post-operatively ${ }^{6,22}$. By contrast in the case series which showed improved outcomes for patients undergoing mitral valve surgery, ECMO was inserted preoperatively allowing for haemodynamic stabilisation of patients before they underwent surgery which would suggest that ECMO may be most useful when inserted pre-operatively alongside inotropes in these patients ${ }^{23}$. However, more work on the potential survival benefit of ECMO use pre-operatively in patients undergoing valvular surgery is needed as only one paper has shown improved outcomes.

\section{ECMO in patients with circulatory arrest}

Deep hypothermic circulatory arrest (DHCA) is a technique utilised during procedures requiring circulatory arrest such as surgery involving the aortic arch for the purpose of organ protection, specifically for cerebral protection, as hypothermia inhibits injury-inducing ischaemic pathways driven by hypoxia ${ }^{24-26}$. DHCA utilises CPB to maintain perfusion to the rest of the body during the procedure but the need for prolonged $\mathrm{CPB}$ comes with its own complications such as coagulopathy and failure to wean from $\mathrm{CPB}$, thereby requiring ECMO for circulatory support.

There is little to no evidence on ECMO use following DHCA in adult patients but there are a few studies that have looked at outcomes in paediatric patients who required ECMO following DHCA as part of the Norwood procedure used for surgical treatment of cardiac defects characterised by the presence of shunted single-right ventricle circulation such as hypoplastic left heart syndrome (HLHS) ${ }^{27-29}$. Analysis of 549 patients with single-right ventricle anomalies enrolled in the Pediatric Heart Network Single Ventricle Reconstruction trial found that post-operative ECMO requirement was a significant indicator for increased 30-day mortality (OR 4.38; 95\% CI 1.76-10.90; $P=0.002$ ) and for hospital mortality (Hazard ratio 3.41; 95\% CI 1.94-5.98; $P$ $<0.001)^{27}$. The study was carried out across paediatric cardiac surgery centres with a large volume of cases annually meaning these findings cannot be generalised to centres with smaller case volumes. However, similar results were shown in a retrospective study analysing risk factors for 1-year mortality in 158 patients that underwent the Norwood procedure which demonstrated that ECMO or VAD support was associated with increased risk of death (OR 17.8; 95\% CI 4.4-71.0; $P<0.001)^{28}$.

Further separate analysis of subjects enrolled in the Single Ventricle Reconstruction trial of 461 patients discharged home after undergoing the Norwood procedure showed that 66 of them (14.3\%) developed heart failure, with 15 of these dying from the heart failure whilst 39 were listed for transplant ${ }^{29}$. The study found that need for ECMO post-operatively, among other factors, was significantly associated with increased risk of developing heart failure within a year post-Norwood procedure (Hazard ratio 5.83; 95\% CI 1.75-19.46; $P$ $=0.004$ ) when compared to those not requiring extracorporeal support. Outcomes for ECMO instituted following DHCA are summarised in Table 5.

The little data that does exist on ECMO following DHCA suggests that those patients requiring postoperative ECMO had an increased mortality both in hospital and following hospital discharge; though 
mortality for PCCS without MCS would be far worse. However, there are very few studies that have been carried out meaning it may not be appropriate to generalise these findings to other populations. The gap in research in this area suggests the need for more to be carried out to see if there is a survival benefit associated with use of ECMO in patients who have undergone DHCA.

\section{Complications of ECMO}

Complications of using ECMO can generally be divided into major and minor complications. They can be classified into various categories; inflammatory response, limb ischemia, anticoagulation-related and infections. Major complications poses a significant threat to the patient's life such as sepsis and intracranial haemorrhage, while minor complications such as bleeding around the cannula sites could occur at an early stage but may progress to more adverse events if appropriate action is not taken to correct them. Table 6 is a summary of such complications.

\section{Inflammatory response}

Contact of the patient's blood with the extracorporeal circuit equipment activates coagulation cascade, complement systems and cytokines, recruiting leukocytes and platelets which then damage endothelial cells on vessels and lead to a hypercoagulable state. Neutrophils are thought to be activated in the oxygenator of the $\mathrm{ECMO}^{30}$. Neutrophilic infiltration into tissues can then cause organ damage ${ }^{31}$. The inflammatory response is similar to that in systemic inflammatory response syndrome (SIRS), causing an increased risk of thrombosis, infections, sepsis and end-organ damage thus worsening patient outcomes.

Prophylactic steroid treatment has shown to reduce postoperative infections, length of stay and reduce infections, however it did not change mortality rates. Mesenchymal stromal cells in animal models have shown to downregulate inflammatory factors and upregulate anti-inflammatory factors and can be promising solutions to be used in patients. Hemadsorption cytokine removal therapy has also shown to reduce CRP and IL-6. However these solutions are novel and more research should be undertaken to ascertain their benefits.

\section{Limb ischemia}

Major vascular complications are common in post-cardiotomy patients after ECMO support mainly due to reduced blood flow which may occur due to various factors such as large cannula ( $>20 \mathrm{Fr}$ ) occluding the vessel, female gender, and peripheral vascular disease like atherosclerosis. Limb amputation may also be necessary in severe and lifesaving cases.

Reperfusion injuries may then occur when blood flow is increased, which may result in rhabdomyolysis, acute kidney injury, multi-organ failure and systemic inflammatory response. Insertion of cannula under fluoroscopic guidance can help significantly reduce limb ischaemia. Frequent monitoring of cannula site will also ensure cannula is not kinked or obstructed by thrombus. A duplex ultrasound can be used to detect blood flow however it is user-dependent and cannot be monitored continuously. Flow monitoring through the ECMO shunt could be feasible for continuous monitoring. Graft interposition can also be done to eliminate limb ischemia.

\section{Anticoagulation-related complications}

Unfractionated heparin is often used for anticoagulation to keep the circulatory system patent to prevent thrombosis formation in the extracorporeal circulation and the patient's circulatory system. Thankfully current ECMO circuits have almost no possibility of thrombus formation. Haemolysis can also occur especially if a thrombus forms in the pump causing mechanical damage to cells resulting in haemolytic anaemia. Heparin-induced thrombocytopenia could also be a cause of clot formation. Intracranial haemorrhage is also a possibility in ECMO patients from excess infusion of anticoagulation and early correction of thrombocytopenia may help reduce risks of occurrence. Other neurological complications such as strokes and cognitive impairment could also result if haemostatic balance is not well controlled.

D-dimers can be monitored daily to look out for any clot formation and to anticipate eminent ECMO failure. Before ECMO initiation, patients should be tested for any coagulation disorders by measuring parameters 
such as antithrombin and fibrinogen levels, which can be corrected to prevent further complications.

\section{Infections}

The Extracorporeal Life Support Organisation (ELSO) approximates the incidence of infection of $13.0 \%$ and $17.5 \%$ in VA-ECMO and VV-ECMO respectively. The prevalence of infection during ECMO is $10-12 \%$, withStaphylococcus aureus, Candida, Enterobacteriaceaeand Pseudomonas aeruginos a being the most common bloodstream infective organisms. Identifying infected patients is difficult due to their inability to mount a pyrexic response possible due to heat loss from ECMO circuit, and leukocytosis is common due to inflammatory response from ECMO treatment itself. Hence, regular cultures could be done if an infection is suspected, especially with prolonged ECMO use.

\section{Future of ECMO in Cardiac surgery}

VA-ECMO is indicated when patients fail to be weaned off CPB or clinically deteriorates significantly during the course of staying on intensive care stay. ECMO provides a longer support for a few days to allow for recovery of the cardiopulmonary system, compared to a few hours of support with CPB. VA-ECMO confers several advantages that helps increasing patient survival and reduce mortality rates. CPB needs a large amount of unfractionated heparin as it utilises a venous reservoir which causes blood to stagnate. CPB also causes blood to pool in the heart chambers and respiratory circulatory system, which result in an increase in activated clotting time.

Whereas, VA-ECMO does not utilise a venous reservoir and is made of shorter tubes. There is reduced occurrence of thrombosis allowing for a reduction in the dosage of unfractionated heparin which can then reduce bleeding complications. Hence, VA-ECMO use in refractory PCCS has been shown to provide significant survival benefits without which mortality would be inevitable. Increased age, renal failure and long use of ECMO support are the most frequently reported adverse prognostic factors .

Over the recent years, the use of ECMO has greatly increased. According to ELSO, the use of adult ECMO increased by about tenfold over the past decade. This is likely following a ground-breaking study, the CESAR trial, which showed a significant increase in survival without severe disability when ECMO was used instead of conventional ventilation. Early initiation of ECMO has been shown to result in higher survival rates and decreasing the dosage of vasoactive drugs by increasing cardiac output and rapidly decreasing arterial lactate levels after cardiovascular surgery. This is shown in a study which compared two groups of patients where the patients with early initiation ECMO failed to be weaned off CPB once, while the patients with delayed ECMO failed to weaned off thrice.This could help provide a guide as to when ECMO should be initiated in patients after cardiac surgery to improve outcomes.

ECMO technology is constantly evolving and improving, with more compact and durable components being introduced in recent times. Wearable ambulatory ECMO has been developed and trialled in patients with severe cardiopulmonary failure awaiting transplant. Compact ECMO is achievable by the use of hollow fibre membranes arranged in stacks with centrifugal pumps. This allows increased patient mobility which helps with rehabilitation and early mobilisation allowing patients to walk and exercise, preventing muscle atrophy allowing shorter recovery time and placing patients in a better physiological state for heart or lung transplant. It also helps to decrease total costs by about $11 \%$ compared to traditional ECMO use. This could soon be the new future of ECMO, which is currently still a complex and bulky piece of equipment resulting in patients being bedbound, slowing recovering.

Unfortunately, ECMO is a very costly form of life support at about $£ 45,000$ per patient. It is also resource intensive, requiring a high level of expertise to use it. An article states that the National Specialist Commissioning Group only funds ECMO to certain specialist centres, restricting its access which is inappropriate and unacceptable considering its great benefits to patients' survival rates. Whereas, in other parts of the world such as the US and Europe, ECMO is routinely used. It is contentious to say whether the UK has too many restrictions to use of ECMO or if the UK is being too prudent. VA ECMO is not commissioned by the National Health Service (NHS) for PCCS and the price of it is forked out by individual hospitals in 
the UK. Most recently, ECMO is being used widely in COVID-19 patients where they may develop cardiac arrhythmias and shock. About $15 \%$ to $30 \%$ of patients with the viral pneumonia developed acute respiratory distress syndrome (ARDS) where the WHO recommendations included ECMO as a part of the management. The role of ECMO for COVID-19 patients is still unclear and is dependent on the mechanism which the virus harms the body. If it causes septic shock and multiorgan failure, the management will likely shift away from ECMO as it may be less helpful in these instances. Although inconclusive, an article brought up concerns about the use of ECMO in COVID-19 patients, suggesting healthcare professionals evaluate the IL-6 concentration and lymphocyte count before and during ECMO, as ECMO increases IL-6 levels and decreases lymphocyte count. More studies should be carried out to determine the usefulness of ECMO in this situation, especially when they need an emergency cardiac surgery.

\section{Summary}

ECMO post cardiac surgery can have detrimental benefit on survival outcomes. There is a survival rate of $30.8 \%$ for patients who underwent ECMO for PCCS, where without ECMO salvage PCCS is inevitably a fatal state for patients. From an ethical viewpoint, one should also consider the possibility of ECMO in prolonging patient's hospital stay without significant improvement or addition to prognostic benefit and therefore careful patient selection should be made through inter-speciality discussions. The patient's best interests should always be considered before initiating any medical intervention with the risks and benefits being weighed out judiciously for each case. Liaising with colleagues will help professionals come to an appropriate decision to prolong life with ECMO or to prevent futile attempts. Risk stratification could also be conducted to determine if prophylactic ECMO would be suitable for use in high-risk patients to help improve survival rates.

\section{Conclusion}

ECMO is a cardiopulmonary support often used in patients who fail to be weaned off CPB, who are likely to have reversible causes. Although there are several complications related to its use, there are steps that can be done to minimise their occurrence. New components and variations of ECMO are being trialled providing better prospects for patients. It has also seen a recent surge of its use in COVID-19 patients. Studies analysed in this article have shown that the circulatory support provided by VA-ECMO improves survival rates for PCCS in patients undergoing cardiac surgery, without which patient mortality rates would be much greater. These benefits of VA-ECMO are especially seen post-CABG and post-valve surgery patients.

\section{References}

1. Makdishi G, Wang I. Extra Corporeal Membrane Oxygenation (ECMO) review of a lifesaving technology. J Thorac Dis 2015;7(7):E166-E174. https://doi.org/10.3978/j.issn.2072-1439.2015.07.17.

2. Allen S, Holena D, McCunn M, et al . A Review of the Fundamental Principles and Evidence Base in the Use of Extracorporeal Membrane Oxygenation (ECMO) in Critically Ill Adult Patients. J Intensive Care Med 2011;26(1):13-26. https://doi.org/10.1177/0885066610384061.

3. Extracorporeal Life Support Organization - ECMO and ECLS $>$ Registry $>$ Statistics $>$ International Summary. https://www.elso.org/Registry/Statistics/InternationalSummary.aspx. Accessed March 20, 2020 .

4. Khorsandi M, Shaikhrezai K, Prasad S, et al . Advanced mechanical circulatory support for postcardiotomy cardiogenic shock: a 20-year outcome analysis in a non-transplant unit. J Cardiothorac Surg 2016;11:29. https://doi.org/10.1186/s13019-016-0430-2.

5. Khorsandi M, Dougherty S, Sinclair A, et al. A 20-year multicentre outcome analysis of salvage mechanical circulatory support for refractory cardiogenic shock after cardiac surgery. J Cardiothorac Surg 2016;8(11):151. https://doi.org/10.1186/s13019-016-0545-5.

6. Rastan AJ, Dege A, Mohr M, et al . Early and late outcomes of 517 consecutive adult patients treated with extracorporeal membrane oxygenation for refractory postcardiotomy cardiogenic shock. $J$ Thorac Cardiovasc Surg 2010;139(2):302-311. https://doi.org/10.1016/j.jtcvs.2009.10.043.

7. Li CL, Wang H, Jia M, et al . The early dynamic behavior of lactate is linked to mortality in post- 
cardiotomy patients with extracorporeal membrane oxygenation support: a retrospective observational study. J Thorac Cardiovasc Surg2015;149(5):1445-50. https://doi.org/10.1016/j.jtcvs.2014.11.052.

8. Hsu P, Chen J, Hong G. Extracorporeal membrane oxygenation for refractory cardiogenic shock after cardiac surgery: predictors of early mortality and outcome from 51 adult patients. Eur J Cardiothorac Surg 2010;37(2):328-333. https://doi.org/10.1016/j.ejcts.2009.07.033.

9. Wu MY, Lin PJ, Lee MY, et al . Using extracorporeal life support to resuscitate adult postcardiotomy cardiogenic shock: treatment strategies and predictors of short-term and midterm survival.Resuscitation 2010;81(9):1111-6. https://doi.org/10.1016/j.resuscitation.2010.04.031.

10. Elsharkawy HA, Li L, Sakr WA, et al . Outcome in Patients Who Require Venoarterial Extracorporeal Membrane Oxygenation Support After Cardiac Surgery. J Cardiothoracic Vasc Anesth2010;24(6):946951. https://doi.org/10.1053/j.jvca.2010.03.020.

11. Rubino A, Costanzo D, Stanszus, et al . Central Veno-Arterial Extracorporeal Membrane Oxygenation (C-VA-ECMO) After Cardiothoracic Surgery: A Single-Center Experience. J Cardiothorac Vasc 2018;32(3):1169-1174. https://doi.org/10.1053/j.jvca.2017.12.003.

12. Slottosch I, Liakopoulos O, Kuhn E, et al . Outcomes after peripheral extracorporeal membrane oxygenation therapy for postcardiotomy cardiogenic shock: a single-center experience. J Surg Res 2013;181(2):47-55. https://doi.org/10.1016/j.jss.2012.07.030.

13. Mikus E, Tripodi A, Calvi S, et al . CentriMag Venoarterial extracorporeal membrane oxygenation support as treatment for patients with refractory Postcardiotomy Cardiogenic shock. ASAIO J2013;59(1):18-23. https://doi.org/10.1097/MAT.0b013e3182768b68.

14. Frenckner B, Broman M, Broomé M. Position of draining venous cannula in extracorporeal membrane oxygenation for respiratory and respiratory/circulatory support in adult patients. Crit Care2018;22:163. https://doi.org/10.1186/s13054-018-2083-0.

15. Rao P, Khalpey Z, Smith R, et al . Venoarterial Extracorporeal Membrane Oxygenation for Cardiogenic Shock and Cardiac Arrest.Circ Heart Fail 2018;11:e004906. https://doi.org/10.1161/CIRCHEARTFAILURE.118.00490:

16. Flécher E, Anselmi A, Corbineau $\mathrm{H}$, et al . Current aspects of extracorporeal membrane oxygenation in a tertiary referral centre: determinants of survival at follow-up. Eur J Cardio-Thoracic Surg 2014;46(4):655-671. https://doi.org/10.1093/ejcts/ezu029.

17. Acker MA. Mechanical circulatory support for patients with acute-fulminant myocarditis. Ann Thorac Surg 2001;71:S73-S76. https://doi.org/10.1016/S0003-4975(00)02628-X.

18. Stewart GC, Giverts MM. Mechanical circulatory support for advanced heart failure: patients and technology in evolution. Circulation 2012;125:1304-15. https://doi.org/10.1161/CIRCULATIONAHA.111.060830.

19. Peek GJ, Mugford M, Tiruvoipati R, et al . Efficacy and economic assessment of conventional ventilatory support versus extracorporeal membrane oxygenation for severe adult respiratory failure (CESAR): a multi-centre randomised controlled trial. Lancet2009;372:1351-63. https://doi.org/10.1016/S01406736(09)61069-2.

20. McLaren G, Combes A, Bartlett RH. Contemporary extracorporeal membrane oxygenation for adult respiratory failure: life support in the new era. Intensive Care Med 2012;38:210-220. https://doi.org/10.1007/s00134011-2439-2.

21. Pipeling MR, Fan E. Therapies for refractory hypoxaemia in acute respiratory distress syndrome. JAMA 2010;304:2521-2527. https://doi.org/10.1001/jama.2010.1752.

22. Onorati F, Quintana E, Dean Z, et al . (2020). Aortic valve replacement for aortic stenosis in low-, intermediate-, and high-risk patients: preliminary results from a prospective multicenter Registry. $J$ Cardiothorac Vasc Anesth, https://doi.org/10.1053/j.jvca.2020.02.045.

23. DiVita M, Visveswaran GK, Naji P, et al . Emergent TandemHeart - ECMO for acute severe mitral regurgitation with cardiogenic shock and hypoxaemia: a case series. Eur Heart $J$ 2020;4(1):1-6. https://doi.org/10.1093/ehjcr/ytz234.

24. Ziganshin BA, Elefteriades JA. Deep hypothermic circulatory arrest.Ann Cardiothorac Surg 2013;2(3):303315. https://doi.org/10.3978/j.issn.2225-319X.2013.01.05.

25. González-Ibarra FP, Varon J, López-Meza EG. Therapeutic hypothermia: critical review of the molecular mechanisms of action. Front Neurol 2011;2:4. https://doi.org/10.3389/fneur.2011.00004. 
26. Conolly S, Arrowsmith JE, Klein AA. Deep hypothermic circulatory arrest. BJA Educ 2010;10(5):138142. https://doi.org/10.1093/bjaceaccp/mkq024.

27. Tabbutt S, Ghanayem N, Ravishankar, et al . Risk factors for hospital morbidity and mortality after the Norwood procedure: A report from the Paediatric Heart Network Single Ventricle Reconstruction trial. J Thorac Cardiovasc Surg 2012;144(4):882-895. https://doi.org/10.1016/j.jtcvs.2012.05.019.

28. Gaynor JW, Mahle WT, Cohen MI, et al. Risk factors for mortality after the Norwood procedure. Eur J Cardiothorac Surg2002;22:82-89. https://doi.org/10.1016/S1010-7940(02)00198-7.

29. Mahle WT, Hu C, Trachtenberg F, et al . Heart Failure Following The Norwood Procedure: An Analysis Of The Single Ventricle Reconstruction Trial. J Heart Lung Transplant2018;37(7):879-885. https://doi.org/10.1016/j.healun.2018.02.009.

30. Rungatscher A, Tessari M, Stranieri C, et al . Oxygenator Is the Main Responsible for Leukocyte Activation in Experimental Model of Extracorporeal Circulation: A Cautionary Tale. Mediat Inflamm2015;2015:484979. https://doi.org/10.1155/2015/484979.

31. Millar JE, Fanning JP, McDonald CI, et al . The inflammatory response to extracorporeal membrane oxygenation (ECMO): A review of the pathophysiology. Crit Care 2016;20(1):1-10. https://doi.org/10.1186/s13054016-1570-4.

32. Dieleman JM, van Paassen J, van Dijk D, et al . Prophylactic corticosteroids for cardiopulmonary bypass in adults. Cochrane Database Syst Rev 2011. https://doi.org/10.1002/14651858.cd005566.pub3.

33. Dieleman JM, Nierich AP, Rosseel PM, et al . Intraoperative high-dose dexamethasone for cardiac surgery: A randomized controlled trial. JAMA 2012;308(17):1761-1767. https://doi.org/10.1001/jama.2012.14144.

34. Qiang Y, Liang G, Yu L. Human amniotic mesenchymal stem cells alleviate lung injury induced by ischemia and reperfusion after cardiopulmonary bypass in dogs. Lab Investig2016;96(5):537-546. https://doi.org/10.1038/labinvest.2016.37.

35. Bruenger F, Kizner L, Weile J, et al . First successful combination of ECMO with cytokine removal therapy in cardiogenic septic shock: A case report. Int J Artif Organs2015;38(2):113-116. https://doi.org/10.5301/ijao.5000382.

36. Yang F, Hou D, Wang J, et al . Vascular complications in adult postcardiotomy cardiogenic shock patients receiving venoarterial extracorporeal membrane oxygenation. Ann Intensive Care2018;8(1):72. https://doi.org/10.1186/s13613-018-0417-3.

37. Bonicolini E, Martucci G, Simons J, et al . Limb ischemia in peripheral veno-arterial extracorporeal membrane oxygenation: A narrative review of incidence, prevention, monitoring, and treatment.Crit Care 2019. https://doi.org/10.1186/s13054-019-2541-3.

38. Girn HRS, Ahilathirunayagam S, Mavor AID, et al . Basic science review: Reperfusion syndrome: Cellular mechanisms of microvascular dysfunction and potential therapeutic strategies. Vasc Endovasc Surg 2007;41(4):277-293. https://doi.org/10.1177/1538574407304510.

39. Jang WJ, Cho YH, Park TK, et al . Fluoroscopy-guided simultaneous distal perfusion as a preventive strategy of limb ischemia in patients undergoing extracorporeal membrane oxygenation. Ann Intensive Care 8 2018;8(1):101. https://doi.org/10.1186/s13613-018-0445-z.

40. Choi MS, Sung K, Cho YH. Clinical pearls of venoarterial extracorporeal membrane oxygenation for cardiogenic shock.Korean Circ J 2019;49(8):657-677. https://doi.org/10.4070/kcj.2019.0188.

41. Badheka A, Stucker SE, Turek JW, et al . Efficacy of Flow Monitoring during ECMO. ASAIO Journal 2017;63(4):496-500. https://doi.org/10.1097/MAT.0000000000000538.

42. Doll N, Kiaii B, Borger M, et al . Five-Year results of 219 consecutive patients treated with extracorporeal membrane oxygenation for refractory postoperative cardiogenic shock. Ann Thorac Surgery 2004;77(1):151-157. https://doi.org/10.1016/S0003-4975(03)01329-8.

43. Kasirajan V, Smedira NG, McCarthy JF, et al . Risk factors for intracranial hemorrhage in adults on extracorporeal membrane oxygenation. Eur J Cardiothorac Surg 1999;15(4):508-514. https://doi.org/10.1016/S10107940(99)00061-5.

44. Thiagarajan RR, Barbaro RP, Rycus PT, et al . Extracorporeal Life Support Organization Registry International Report 2016.ASAIO Journal 2017;63(1):60-67. https://doi.org/10.1097/MAT.0000000000000475.

45. Biffi S, di Bella S, Scaravilli V, et al . Infections during extracorporeal membrane oxygenation: 
epidemiology, risk factors, pathogenesis and prevention. Int Antimicrob Agents2017;50(1):9-16. https://doi.org/10.1016/j.ijantimicag.2017.02.025.

46. Khorsandi M, Dougherty S, Bouamra O, et a l. Extra-corporeal membrane oxygenation for refractory cardiogenic shock after adult cardiac surgery: A systematic review and meta-analysis. $J$ Cardiothorac Surg 2017;12(1):55. https://doi.org/10.1186/s13019-017-0618-0.

47. Ge M, Pan T, Wang JX, et al . Outcomes of early versus delayed initiation of extracorporeal life support in cardiac surgery. J Cardiothorac Surg 2019;14(1):129. https://doi.org/10.1186/s13019-019-0950-7.

48. Madhani SP, Frankowski BJ, Burgreen GW, et al . In vitro and in vivo evaluation of a novel integrated wearable artificial lung.J Heart Lung Transplant 2017;36(7):806-811. https://doi.org/10.1016/j.healun.2017.02.025.

49. Wu ZJ, Zhang T, Bianchi G, et al . Thirty-day in-vivo performance of a wearable artificial pump-lung for ambulatory respiratory support. Ann Thorac Surg 2012;93(1):274-281. https://doi.org/10.1016/j.athoracsur.2011.08.076

50. Bain JC, Turner DA, Rehder KJ, et al . Economic outcomes of extracorporeal membrane oxygenation with and without ambulation as a bridge to lung transplantation. Respir Care 2016;61(1):1-7. https://doi.org/10.4187/respcare.03729.

51. Scottish Government T. Scottish Government Health Directorates The Provision of Non-H1N1 Adult Respiratory Extra Corporeal Membrane Oxygenation (ECMO) in the Medium and Longer Term for Scotland ECMO Expert Group Report and Recommendations December 20092 CONTENTS.

52. Westaby S, Taggart D. Inappropriate restrictions on life saving technology. Heart 2012;98(15):1117-9. https://doi.org/10.1136/heartjnl-2011-301365.

53. Sedgwick C, Gati S, Sharma S. Extra corporeal membrane oxygenation (ECMO) devices in all heart attack centres in the UK?. Br J Cardiol 2012;19:102-03. https://doi.org/10.5837/bjc.2012.021.

54. Westaby S, de Silva R, George S, et al . Lessons learnt from a tragic loss - But will things improve? Br J Cardiol2012;19:141-3. https://doi.org/10.5837/bjc.2012.027.

55. Clinical management of severe acute respiratory infection when novel coronavirus (nCoV) infection is suspected. https://www.who.int/publications-detail/clinical-management-of-severe-acute-respiratory-infectionwhen-novel-coronavirus-(ncov)-infection-is-suspected. Accessed March 20, 2020.

56. MacLaren G, Fisher D, Brodie D. Preparing for the Most Critically Ill Patients With COVID-19: The Potential Role of Extracorporeal Membrane Oxygenation. JAMA 2020. https://doi.org/10.1001/jama.2020.2342.

57. Henry BM. COVID-19, ECMO, and lymphopenia: a word of caution.Lancet Respir Med 2020. https://doi.org/10.1016/S 2600(20)30119-3.

\section{Figure legends:}

Figure 1: Schematic diagram illustrating double cannulation Veno-venous (VV) ECMO ${ }^{14}$. a . Draining from the SVC/RA then passed through an extracorporeal membrane oxygenator (OX ) before being infused into femoral vein. $\mathbf{b}$. Blood is removed from the IVC then passed through an oxygenator before being infused to the right atrium.

Figure 2: Schematic figure showing central and peripheral Veno-arterial extracorporeal membrane oxygenation (VA-ECMO $)^{15}$.A . Peripheral VA-ECMO with blood extracted via the femoral vein and infused through the femoral artery (i.e. femoro-femoral configuration). B . Central VA-ECMO with blood extracted from right atrium and infused via ascending aorta.

\section{Hosted file}

Tables.docx available at https://authorea.com/users/312806/articles/443417-the-use-of-extracorporeal-membrane-oxygenation-post-cardiotomy-the-survival-benefits 

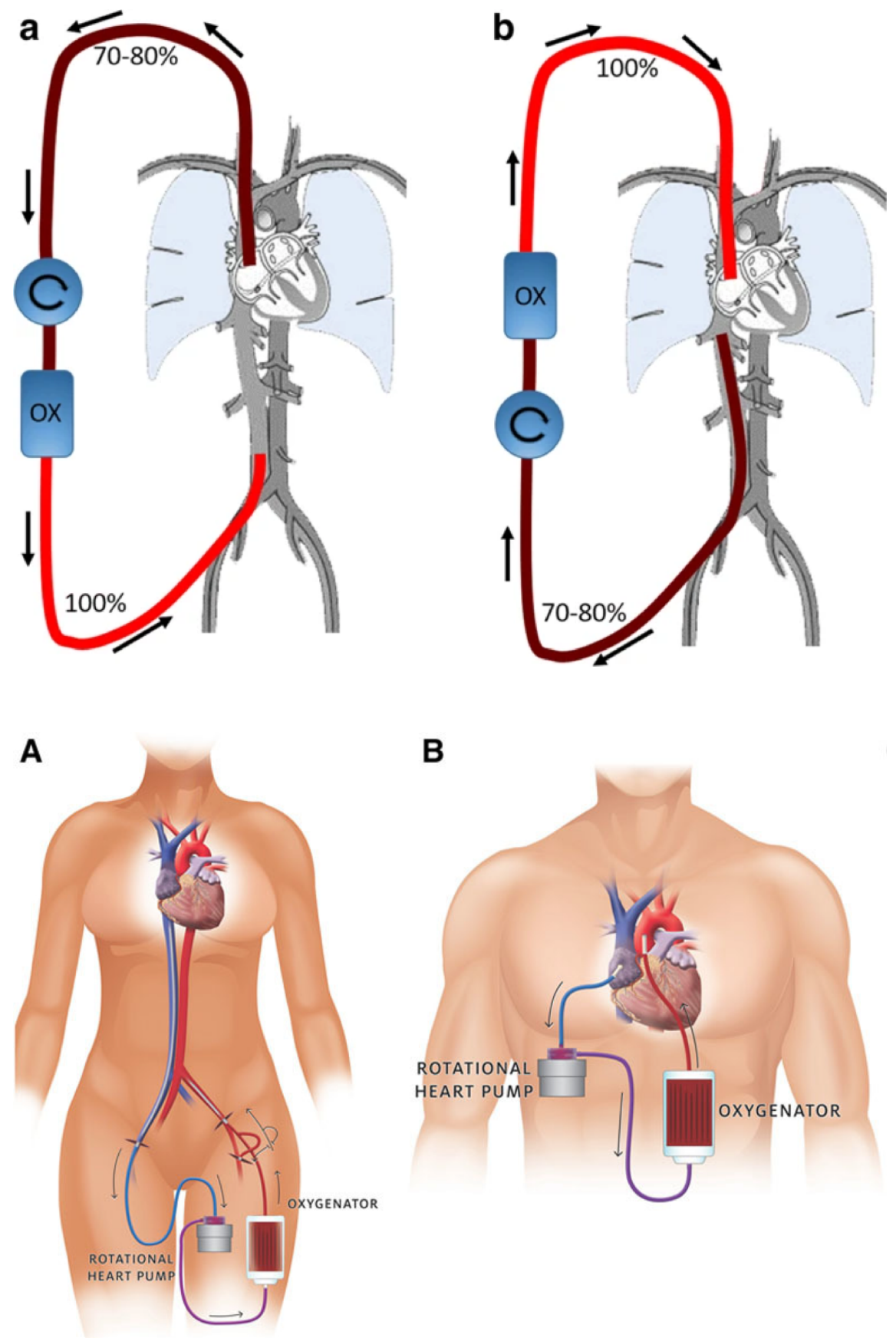